\title{
EVIDENCE FOR HIGH-FREQUENCY QPOs WITH A 3:2 FREQUENCY RATIO FROM A 5000 SOLAR MASS BLACK HOLE
}

\author{
Dheeraj R. Pasham ${ }^{1,2,3}$, S. Bradley Cenko ${ }^{1,2,3}$, Abderahmen Zoghbi, ${ }^{4,5}$, Richard F. Mushotzky ${ }^{1,3}$, \\ Jon Miller ${ }^{4}$, And Francesco Tombesi ${ }^{1,2,3}$ \\ ${ }^{1}$ Astrophysics Science Division, NASA's Goddard Space Flight Center, Greenbelt, MD 20771, USA; dheerajrangareddy.pasham@nasa.gov, brad.cenko@nasa.gov \\ ${ }^{2}$ Astronomy Department, University of Maryland, College Park, MD 20742, USA; ftombesi@astro.umd.edu, richard@astro.umd.edu \\ ${ }^{3}$ Joint Space-Science Institute, University of Maryland, College Park, MD 20742, USA \\ ${ }^{4}$ Department of Astronomy, University of Michigan, Ann Arbor, MI 48109-1042, USA; abzoghbi@umich.edu, jonmm@umich.edu \\ ${ }^{5}$ Cahill Center for Astrophysics, California Institute of Technology, Pasadena, CA 91125, USA \\ Received 2015 June 25; accepted 2015 August 31; published 2015 September 21
}

\begin{abstract}
Following the discovery of 3:2 resonance quasi-periodic oscillations (QPOs) in M82X-1, we have constructed power density spectra (PDS) of all 15 (sufficiently long) XMM-Newton observations of the ultraluminous X-ray source NGC $1313 \mathrm{X}-1\left(L_{\mathrm{X}} \approx 2 \times 10^{40} \mathrm{erg} \mathrm{s}^{-1}\right)$. We detect a strong QPO at a frequency of $0.29 \pm 0.01 \mathrm{~Hz}$ in data obtained on 2012 December 16. Subsequent searching of all the remaining observations for a 3:2/2:3 frequency pair revealed a feature at $0.46 \pm 0.02 \mathrm{~Hz}$ on 2003 December 13 (frequency ratio of $1.59 \pm 0.09$ ). The global significance of the $0.29 \mathrm{~Hz}$ feature considering all frequencies between 0.1 and $4 \mathrm{~Hz}$ is $>3.5 \sigma$. The significance of the $0.46 \pm 0.02 \mathrm{~Hz}$ QPO is $>3.5 \sigma$ for a search at $2 / 3$ and $3 / 2$ of $0.29 \mathrm{~Hz}$. We also detect lower-frequency QPOs $(32.9 \pm 2.6$ and $79.7 \pm 1.2 \mathrm{mHz})$. All the QPOs are superimposed on a continuum consisting of flat-topped, bandlimited noise, breaking into a power law at a frequency of $16 \pm 3 \mathrm{mHz}$ and white noise at $\gtrsim 0.1 \mathrm{~Hz}$. NGC $1313 \mathrm{X}$ 1's PDS is analogous to stellar-mass black holes' (StMBHs) PDS in the so-called steep power-law state, but with the respective frequencies (both QPOs and break frequencies) scaled down by a factor of $\sim 1000$. Using the inverse mass-to-high-frequency QPO scaling of StMBHs, we estimate NGC 1313 X-1's black hole mass to be $5000 \pm$ $1300 M_{\odot}$, consistent with an inference from the scaling of the break frequency. However, the implied Eddington ratio, $L_{\mathrm{Edd}}>0.03 \pm 0.01$, is significantly lower compared to that of StMBHs in the steep power-law state $\left(L_{\mathrm{Edd}}\right.$ $\gtrsim 0.2$ ).
\end{abstract}

Key words: black hole physics - methods: data analysis - X-rays: binaries - X-rays: individual (NGC 1313 X-1)

\section{INTRODUCTION}

Compact accreting X-ray sources in nearby galaxies with luminosities exceeding $10^{39} \mathrm{erg} \mathrm{s}^{-1}$ are referred to as ultraluminous X-ray sources (ULXs). Current evidence suggests that ULXs might be a mixed bag of compact objects including stellar-mass black holes (StMBHs: 3-25 $M_{\odot}$ ) powered by super-Eddington accretion (e.g., King et al. 2001; Begelman 2002; Gladstone et al. 2009), intermediate-mass black holes (IMBHs: a few $\times(100-1000) M_{\odot}$; Kaaret et al. 2001, 2006; Matsumoto et al. 2001; Miller et al. 2004; Farrell et al. 2009; Pasham et al. 2014; Mezcua et al. 2015), and neutron stars (Bachetti et al. 2014).

One of the biggest challenges in understanding ULXs is to estimate their compact object masses. Because their optical counterparts are faint (V-band magnitudes of 22-24; e.g., Tao et al. 2011; Gladstone et al. 2013), Doppler tracking their optical counterparts to derive their mass functions-as done for Galactic StMBHs - has been extremely challenging (e.g., Roberts et al. 2011; Cseh et al. 2013). However, in a few ULXs, such optical measurements have yielded mass constraints that suggest lower-mass black holes $\left(\lesssim 30 M_{\odot}\right.$; Liu et al. 2013; Motch et al. 2014).

It has been suggested that the detection of the 3:2 frequency ratio high-frequency quasi-periodic oscillations (QPOs) can resolve the ULX mass problem (Abramowicz et al. 2004). StMBH high-frequency QPOs (frequency range of 100-450 Hz; McClintock \& Remillard 2006; Belloni et al. 2012) that appear in a $3: 2$ frequency ratio scale inversely with the black hole mass. Moreover, it has been demonstrated that the power density spectra (PDS) of both the stellar-mass and the supermassive black holes are qualitatively similar. The PDS break timescales of both simply scale with the black hole mass after accounting for the differences in the accretion efficiency between sources (e.g., McHardy et al. 2006; Körding et al. 2007). One can also use this break timescale to estimate black hole masses. Thus, under this black hole variability unification paradigm, 3:2 high-frequency QPO analogs of StMBHs should also be detectable from IMBHs, but with centroid frequencies scaled down according to their respective black hole masses (Vaughan \& Uttley 2005). For example, a few $1000 M_{\odot}$ IMBH should exhibit high-frequency QPOs with centroid frequencies in the range of a fraction of $\mathrm{Hz}$. In fact, such 3:2 ratio QPOs have already been detected from the ULX M82X-1. In that source, the two QPOs $(3.3$ and $5 \mathrm{~Hz}$ ) allowed Pasham et al. (2014) to estimate its black hole mass to be 428 $\pm 105 M_{\odot}$. Here, we report evidence for a second such highfrequency pair from another ULX, NGC 1313 X-1.

\section{XMM-Newton DATA}

As of the writing of this paper, 22 of the 24 XMM-Newton observations of NGC 1313 are public. The three brightest $\mathrm{X}$-ray sources in this field, the two ULXs, NGC $1313 \mathrm{X}-1$ (hereafter, $\mathrm{X}-1$ ) and $\mathrm{X}-2$ and the $\mathrm{X}$-ray bright supernova SN1978K are well separated in the XMM images (see Figure 1). Previous energy spectral studies of X-1 suggest it may host an IMBH with a mass of $\sim 1000 M_{\odot}$ (e.g., Miller et al. 2003, 2013). Given the frequency range we are interested in, we used data primarily from EPIC-pn, utilizing events in the entire 


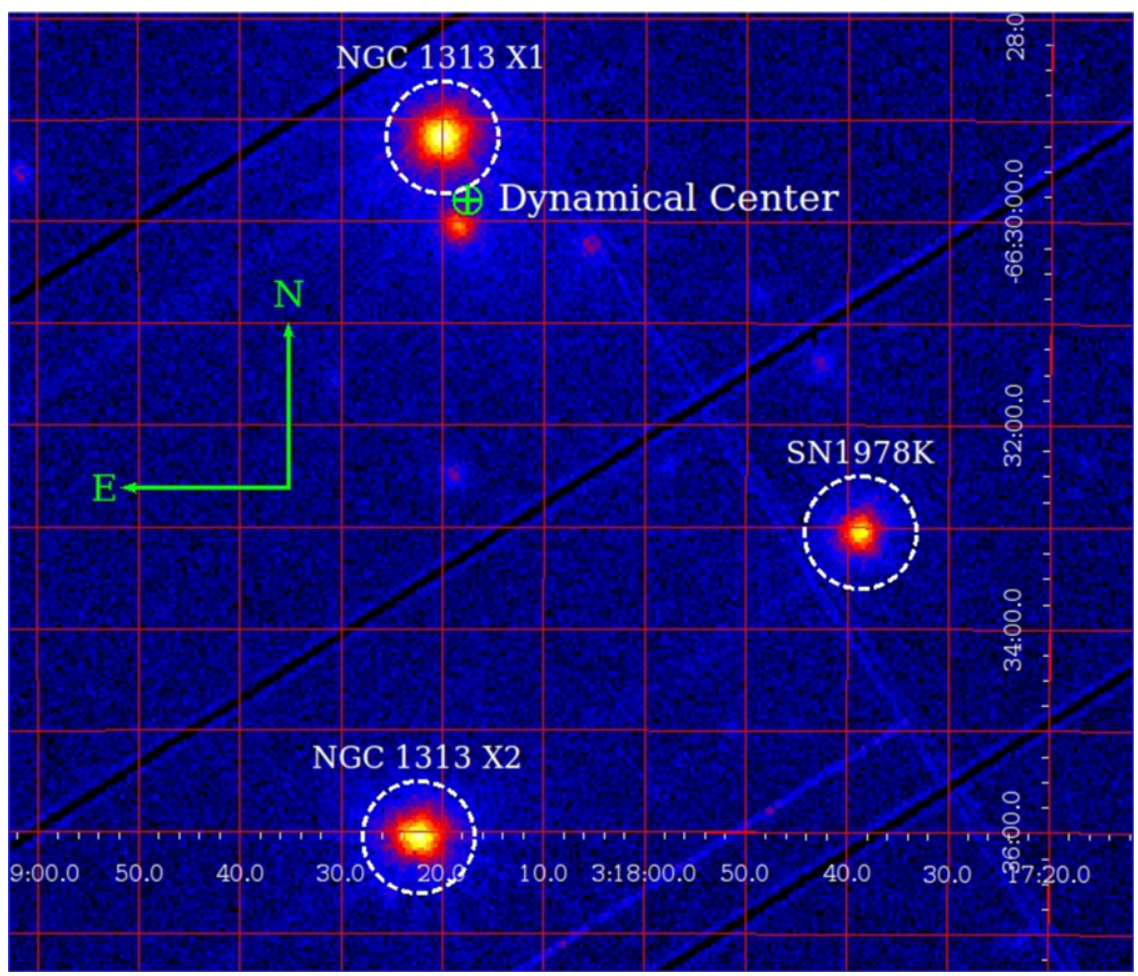

Figure 1. XMM-Newton/EPIC-pn X-ray (0.3-10 keV) image of NGC 1313, from observation 0693850501, produced with DS9. The dashed circles have a radius of $33^{\prime \prime}$ and indicate the size of our typical source extraction region. The dynamical center of the host galaxy, shown as an encircled cross, is based on H i maps by Ryder et al. (1995). The north and the east arrows are each $100^{\prime \prime}$ long.

0.3-10.0 keV band pass. Both the pn and the MOS detectors were operated in the so-called full-frame mode during all the observations. While pn's full-frame data mode offers a time resolution of $73.4 \mathrm{~ms}$, i.e., a Nyquist frequency of $6.82 \mathrm{~Hz}$, MOS data are limited to a Nyquist frequency of only $0.19 \mathrm{~Hz}$.

\section{ANALYSIS}

We reduced all the observations using the standard data reduction procedures and removed data sets that were severely affected by background flaring. This preliminary screening left us with 15 observations (Table 1). Source events were extracted from a circular region with a radius of 33" (dashed circle around X-1 in Figure 1) when X-1 was clear of a CCD gap. When X-1 was close to or on a CCD gap we extracted events from a smaller region of radius $25^{\prime \prime}$ excluding the CCD gap. Circular background regions of the same radius, free of any point sources, were chosen away from the source's readout column and as close to the telescope pointing as possible.

\subsection{Results: Timing}

In order to assess X-1's variability, we first extracted the source and the background light curves from each of the 15 observations. Background flaring was prominent for only brief durations in some observations. We constructed good time intervals (GTIs) accounting for both the background flares and times when the detector was turned off. Figure 2 contains sample background-subtracted X-ray light curves (black) and their respective backgrounds (red) from all the observations whose power spectra are described in this article.

Using the GTIs shown in Figure 2, we constructed a Leahy normalized (Poisson noise level of 2; Leahy et al. 1983) PDS of $\mathrm{X}-1$ from each of the individual observations. All the power spectra were sampled only up to $4 \mathrm{~Hz}$, a value safely below the Nyquist frequency of $6.82 \mathrm{~Hz}$, in order to avoid any aliasing affects. We started our timing analysis with the three longest observations (obsIDs: 0405090101, 0693850501, and 0693851201) during which X-1 was positioned on-axis, giving the best sensitivity for detecting QPOs.

\subsubsection{ObsID 0405090101}

The top panel of Figure 3 shows the combined EPIC (pn +MOS) PDS from obsID 0405090101-sampled in the frequency range from 0.0022 to $4 \mathrm{~Hz}$. We first extracted a pn-only PDS and found a QPO-like feature at $80 \mathrm{mHz}$ (see top left panel of Figure 1), a frequency well below the MOS detectors' Nyquist frequency. Therefore, in order to improve the signal, we used pn+MOS data in this single instance. It is evident that the overall shape of the power spectrum is flattopped at the lowest frequencies, breaking into a power law, and white noise at the highest frequencies. Two broad QPO features at centroid frequencies of roughly 30 and $80 \mathrm{mHz}$ are also apparent. We modeled the continuum with a constant plus a bending power-law model ${ }^{6}$ similar to other ULXs (e.g., Dheeraj \& Strohmayer 2012; Pasham \& Strohmayer 2013), and StMBHs in the steep power-law state (McClintock \& Remillard 2006). This gave a best-fit $\chi^{2}$ of 419 for 252 degrees

$$
\text { Power }=C+\frac{N \times \nu^{-\alpha}}{\left[1+\left(\frac{\nu}{\nu_{\text {bend }}}\right)^{\beta-\alpha}\right]}
$$

where $\mathrm{C}, \mathrm{N}$, and $\nu_{\text {bend }}$ are the Poisson noise level, the normalization of the bending power law, and the bending frequency, respectively, while $\alpha$ and $\beta$ are the power-law indices below and above the bending frequency, respectively. 
Table 1

Summary of the XMM-Newton Observations of NGC 1313 X-1

\begin{tabular}{|c|c|c|c|c|c|}
\hline$\overline{\text { ObsID }}$ & Date (UTC) ${ }^{\mathrm{a}}$ & $\begin{array}{l}\text { Observation } \\
\text { Time }(\mathrm{ks})^{\mathrm{b}}\end{array}$ & $\begin{array}{l}\text { Count rate } \\
\left(\text { counts s }^{-1}\right)\end{array}$ & $\begin{array}{c}\text { Effective } \\
\text { Exposure }^{\mathrm{d}}(\mathrm{ks})\end{array}$ & $\begin{array}{c}\text { Number of } \\
\text { GTIs }>7 \mathrm{ks}\end{array}$ \\
\hline 0106860101 & 2000 Oct 17 & 42.4 & $0.73 \pm 0.005$ & 31.6 & 1 \\
\hline 0150280301 & 2003 Dec 21 & 16.2 & $1.02 \pm 0.01$ & 9.7 & 1 \\
\hline 0150280401 & 2003 Dec 23 & 20.9 & $0.91 \pm 0.008$ & 10.5 & 1 \\
\hline 0150280501 & 2003 Dec 25 & 21.4 & $0.70 \pm 0.007$ & 9.8 & 1 \\
\hline 0150280601 & 2004 Jan 08 & 53.2 & $0.79 \pm 0.007$ & 12.4 & 1 \\
\hline 0150281101 & 2004 Jan 16 & 8.9 & $0.87 \pm 0.01$ & 7.0 & 1 \\
\hline 0205230301 & 2004 Jun 05 & 11.9 & $1.27 \pm 0.01$ & 10.0 & 1 \\
\hline 0205230401 & 2004 Aug 23 & 18.0 & $0.63 \pm 0.006$ & 14.9 & 1 \\
\hline 0205230501 & 2004 Nov 23 & 16.0 & $0.26 \pm 0.004$ & 14.0 & 1 \\
\hline 0205230601 & 2005 Feb 07 & 14.3 & $0.57 \pm 0.007$ & 12.4 & 1 \\
\hline 0301860101 & 2006 Mar 06 & 21.8 & $1.11 \pm 0.008$ & 19.9 & 1 \\
\hline 0405090101 & 2006 Oct 15 & 123.1 & $0.70 \pm 0.002$ & 121.1 & 3 \\
\hline 0693850501 & 2012 Dec 16 & 125.2 & $0.83 \pm 0.003$ & 123.0 & 4 \\
\hline 0693851201 & 2012 Dec 22 & 125.2 & $0.85 \pm 0.003$ & 123.0 & 4 \\
\hline 0722650101 & 2013 Jun 08 & 30.7 & $0.71 \pm 0.005$ & 28.8 & 2 \\
\hline
\end{tabular}

Notes.

${ }^{a}$ Coordinated universal time.

b Total observation time in ks.

c Average EPIC-pn 0.3-10 keV count rate of NGC 1313 X-1. Note, however, that X-1 was not always on-axis.

${ }^{\mathrm{d}}$ After accounting for flaring background and instrumental good time intervals.

of freedom (dof). We then added a Lorentzian component to model the QPO at $80 \mathrm{mHz}$. This improved the $\chi^{2}$ by 89 , i.e., $\chi^{2}$ of 330 for 249 dof. Using the F-test, this corresponds to a significance of $8 \times 10^{-13}$ or $>7 \sigma$. The best-fit QPO has a centroid frequency, width, normalization, and an rms amplitude of $79.7 \pm 1.2 \mathrm{mHz}, 14.5 \pm 3.4 \mathrm{mHz}, 0.57 \pm 0.09$, and $10.8 \pm$ $2.4 \%$, respectively. Adding a second QPO improved the $\chi^{2}$ by 40 (290 for 246 dof), which corresponds to an F-test probability of $6 \times 10^{-7}(>4.9 \sigma)$. The best-fit centroid frequency, width, normalization, and rms amplitude of the second QPO were $32.9 \pm 2.6 \mathrm{mHz}, 18.2 \pm 7.8,0.34 \pm 0.08$, and $9.4 \pm 3.9 \%$, respectively.

However, Protassov et al. (2002) pointed out the problems with applying the F-test in additive models. Therefore, in order to estimate the QPO significances independent of the F-test, we employed a rigorous Monte Carlo approach as follows.

1. We estimated the baseline bending power law plus a constant model parameters along with their uncertainties and then randomly sampled $N=1.8 \times 10^{6}$ model parameter sets from within the best-fit parameter error bars (assuming normal distribution).

2. For each of these parameter sets, we simulated a light curve of the same length as the observed one following the algorithm described in Timmer \& Koenig (1995) and then extracted a PDS from this simulated light curve.

3. After binning these PDS in the same way as the original PDS, we modeled them with a bending power law. We then added a QPO to this model, with the QPO frequency constrained to lie between 0.01 and $1 \mathrm{~Hz}$. The maximum improvement in $\chi^{2}\left(\Delta \chi_{\max }^{2}\right)$ was recorded from each simulated PDS.

4. The significance of the $80 \mathrm{mHz}$ QPO was estimated as $\left.1-N_{\left(\Delta \chi_{\max }^{2}>\Delta \chi_{\mathrm{obs}}^{2}\right)}\right) / N$, where $N_{\left(\Delta \chi_{\max }^{2}>\Delta \chi_{\mathrm{obs}}^{2}\right)}$ is the number of simulated $\Delta \chi_{\max }^{2}$ values greater than the observed $\Delta \chi_{\mathrm{obs}}^{2}$. For estimating the significance of the $30 \mathrm{mHz}$ QPO, we assumed the baseline model to be the best-fit continuum plus $80 \mathrm{mHz}$ QPO of the observed PDS, and recorded the maximum $\Delta \chi^{2}$ values by adding an additional QPO to this base model.

This methodology is similar to estimating the significances of spectral lines in energy spectra (e.g., Tombesi et al. 2010; Zoghbi et al. 2015). For the $80 \mathrm{mHz}$ feature we ran $1.8 \times 10^{6}$ simulations. The maximum $\Delta \chi^{2}$ was 25 , which is much lower than the observed value of 89 . Thus, we conclude that the $80 \mathrm{mHz}$ feature is significant at least at the $5 \sigma$ level. We ran 200,000 simulations to test the significance of the $30 \mathrm{mHz}$ 

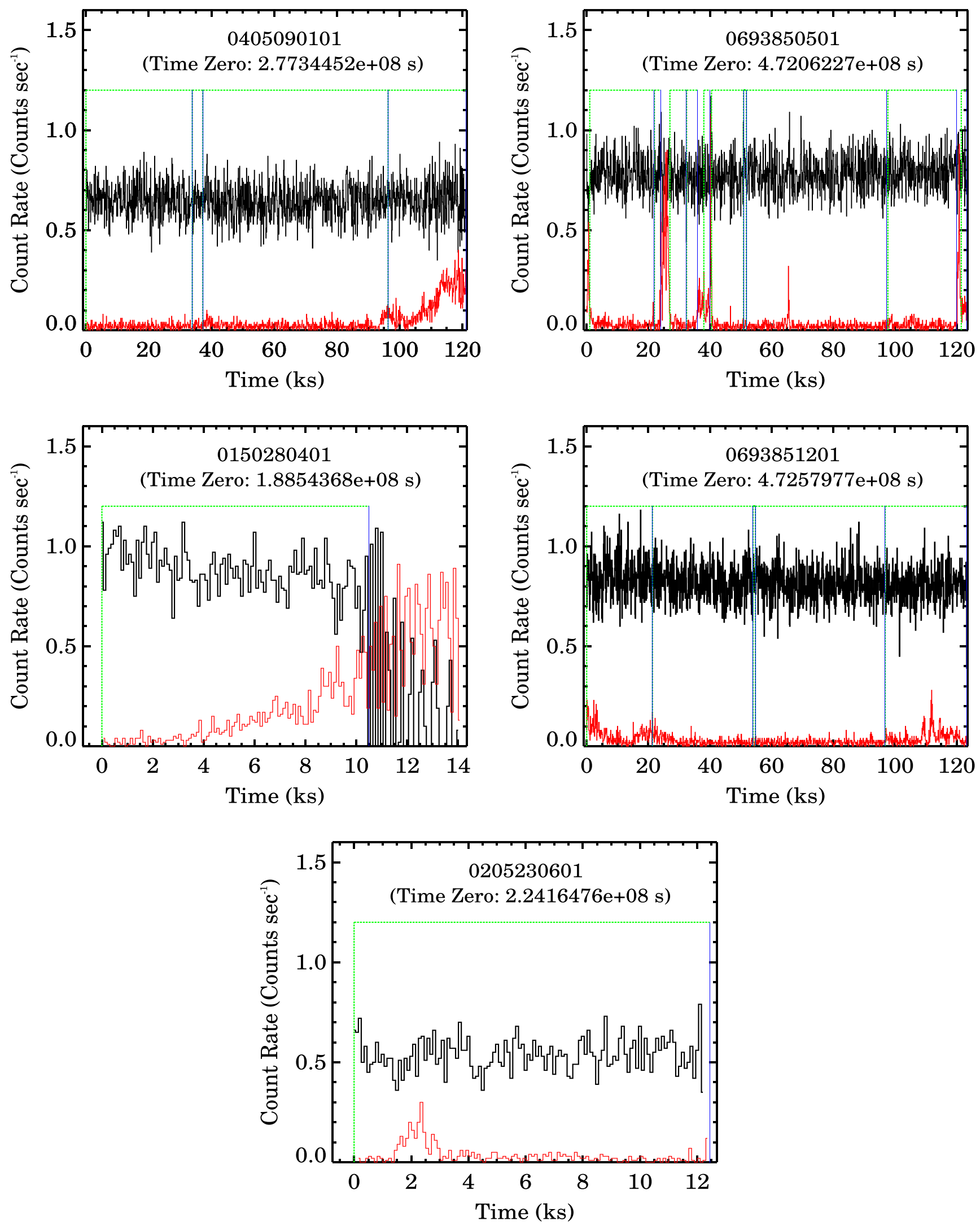

Figure 2. Background-subtracted EPIC-pn X-ray (0.3-10 keV) light curves of NGC 1313 X-1 (black) and their backgrounds (red) during five different XMM-Newton observations. The PDS from these data sets are shown in Figures 3 and 4. For each panel, their observation ID and time zero in seconds since 1998.0 TT are indicated at the top. Also shown are the good time intervals (GTIs) with durations longer than $500 \mathrm{~s}$. A dashed vertical green line and a solid vertical blue line mark the beginning and end of a GTI, respectively. 

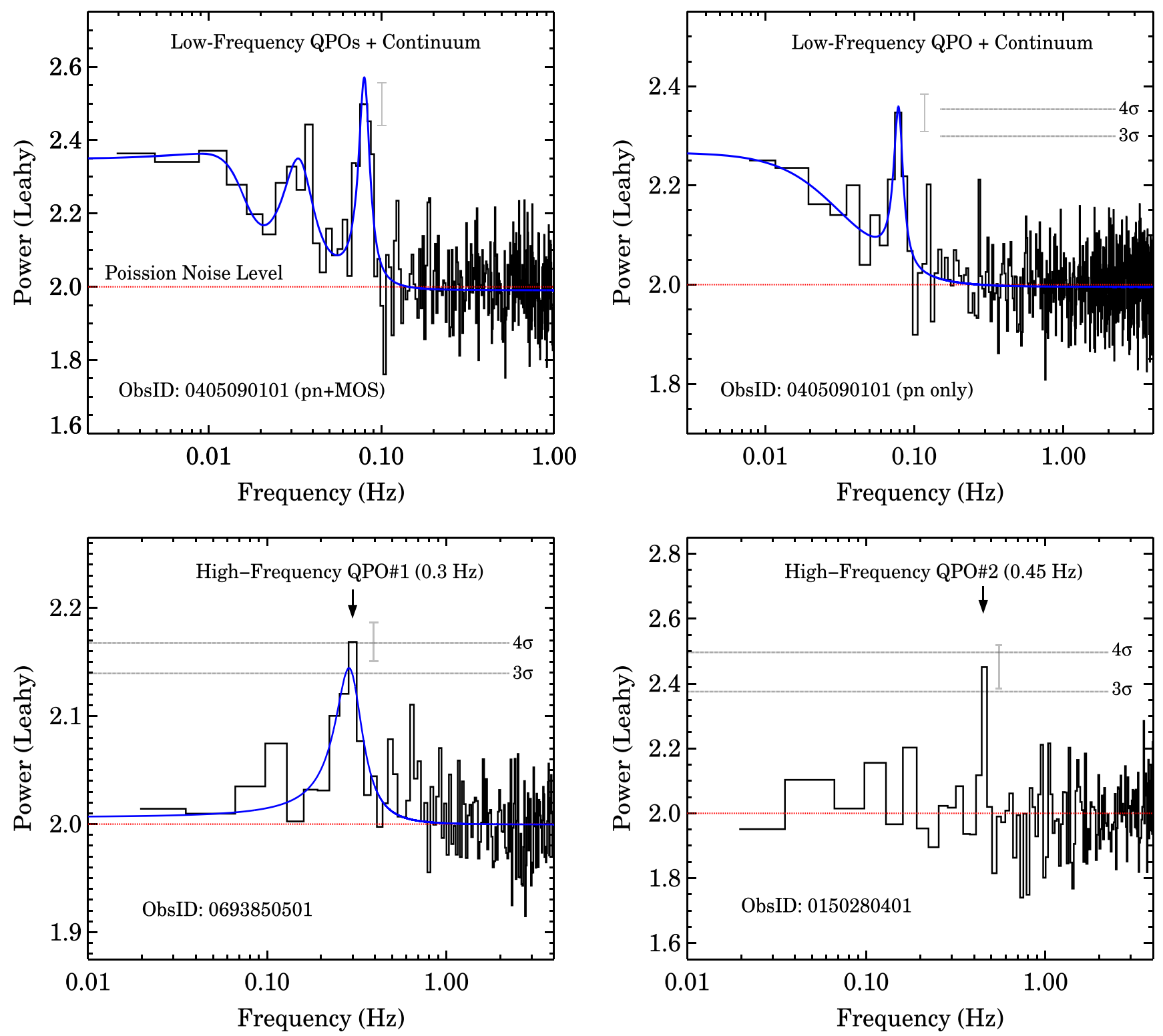

Figure 3. XMM-Newton/EPIC (0.3-10.0 keV) PDS of NGC 1313 X-1 from three different observations. Top left panel: pn+MOS PDS from observation 0405090101 (frequency resolution of $3.9 \times 10^{-3} \mathrm{~Hz}$ ). The continuum was modeled with a constant plus a bending power law, while the QPOs at $30 \mathrm{mHz}(Q$ value: $\nu / \Delta \nu \approx 2)$ and $80 \mathrm{mHZ}(Q$ value $\approx 5.5)$ were modeled with Lorentzians. The solid blue line is the best-fit model. The PDS was obtained by averaging 235 PDS constructed from $512 \mathrm{~s}$ light curve segments binned at $1 / 8 \mathrm{~s}$. Top right panel: pn-only PDS from observation 0405090101 (frequency resolution of $7.8 \times 10^{-3} \mathrm{~Hz}$ ). The 3 and the $4 \sigma$ contours for $\gtrsim 0.1 \mathrm{~Hz}$ (considering all frequency bins between 0.1 and $4 \mathrm{~Hz}$ ) and the best-fit bending power-law model with a QPO (blue) are also shown. Bottom left panel: EPIC-pn PDS (frequency resolution of $0.03125 \mathrm{~Hz}$ ) from observation 0693850501 showing a strong QPO feature at $0.29 \pm 0.01 \mathrm{~Hz}(Q$ value $=2.2)$. Also shown are the 3 and the $4 \sigma$ significance contours considering all the frequency bins (trials) within $0.1 \mathrm{and} 4 \mathrm{~Hz}$ of this observation. While the F-test suggests a significance of $>6 \sigma$, full Monte Carlo simulations imply a lower limit of $5 \sigma$. Bottom right panel: EPIC-pn PDS from observation 0150280401 showing a power spectral feature at $0.46 \pm 0.02 \mathrm{~Hz}(Q>14)$, consistent with $3 / 2$ of $0.29 \mathrm{~Hz}$. This PDS was constructed from $128 \mathrm{~s}$ segments binned at $1 / 8 \mathrm{~s}$. The 3 and the $4 \sigma$ contours take into account four bins (two at $3 / 2$ and $2 / 3$ of $0.3 \mathrm{~Hz}$ and each $0.03125 \mathrm{~Hz}$ wide). In all three cases, the Poisson noise level is marked by a dashed red line and the $1 \sigma$ error bars of the highest bins of the QPOs are indicated in gray.

feature and found that one run exceeded the observed $\Delta \chi^{2}$ of 40. Thus, we conclude its significance is $1-(1 / 200,000)$ or $\approx 4.4 \sigma$.

\subsubsection{ObsID 0693850501}

The PDS from observation 0693850501 exhibited a strong feature at roughly $0.3 \mathrm{~Hz}$ (bottom left panel of Figure 3). The continuum looks flat because we only sampled up to roughly $0.02 \mathrm{~Hz}$. A flat-topped followed by a power-law-like continuum can be seen when we sample up to $0.001 \mathrm{~Hz}$ using longer light curve segments. Modeling the continuum with a constant yielded a best-fit $\chi^{2}$ of 186 for 127 dof. Adding a Lorentzian component improved the $\chi^{2}$ by 56 with an addition of three parameters ( $\chi^{2}$ of 130 for 124 dof). This corresponds to an F-test probability of $1.1 \times 10^{-9}$ or $>6 \sigma$. Using the Monte Carlo approach to test the significance, with $1.8 \times 10^{6}$ simulations, we find a lower limit of $5 \sigma$. For the Monte Carlo simulations, we modeled each of the simulated PDS with a constant and a constant plus a Lorentzian model and recorded the maximum improvement in the $\Delta \chi^{2}$. Out of the $1.8 \times 10^{6}$ simulations, the maximum $\Delta \chi^{2}$ improvement was 27 , a value much lower than the observed $\Delta \chi^{2}$ improvement of 56 . The 
best-fit centroid frequency, width, normalization, and the rms of the QPO were $0.29 \pm 0.01 \mathrm{~Hz}, 0.13 \pm 0.04 \mathrm{~Hz}, 0.14 \pm 0.03$, and $19.0 \pm 5.0 \%$, respectively.

We also estimated the significance using another independent method. First we rescaled the PDS (rescaling factor of 1.01 and the rescaled power at $0.29 \mathrm{~Hz}$ - in the highest bin-is 2.173 ) so that the local mean around $0.5 \mathrm{~Hz}$ is equal to 2, the value expected from a purely Poisson (white noise) process. We then computed the probability with $3 \sigma$ and the $4 \sigma$ confidence of obtaining a power that is at or higher than some threshold $P_{*}$, Probability $\left(P>P_{*}\right)=N_{\text {trials }} \times Q\left(P_{*} \times 898 \times 4 \mid 2 \times 898 \times 4\right)$, where $Q\left(P_{*} \times 898 \times 4 \mid 2 \times 898 \times 4\right)$ is the probability of obtaining a $\chi^{2}$ value of $P_{*} \times 898 \times 4$ or higher from a $\chi^{2}$ distribution with $2 \times 898 \times 4$ dof. We used this $\chi^{2}$ distribution because we averaged in frequency by a factor of four and averaged 898 individual power spectra each derived from $128 \mathrm{~s}$ light curve segments. $N_{\text {trials }}$ account for the total number of trials (frequency bins within $0.1-4 \mathrm{~Hz}$ ). The confidence contours are marked by horizontal dotted lines in the bottom left panel of Figure 3. Clearly, the highest bin in the $0.3 \mathrm{~Hz}$ QPO is significant at greater than the $4 \sigma$ level.

After establishing the lower-frequency continuum and QPOs and the $0.3 \mathrm{~Hz}$ QPO, we searched for a signal at $2 / 3$ and $3 / 2$ of $0.3 \mathrm{~Hz}$ separately from all the GTIs longer than $7 \mathrm{ks}$. The PDS from the fourth GTI (exposure $\approx 22 \mathrm{ks}$ ) of this observation (top panel of Figure 4) showed evidence for excess power at $0.44 \pm 0.06 \mathrm{~Hz}$, a value consistent with $3 / 2$ of $0.3 \mathrm{~Hz}$. This feature is significant at the $7.6 \times 10^{-4}$ level (the significance is $1 \times 10^{-2}$ if all frequencies were searched). We estimated the significance of this feature as follows. First, we estimated the probability of detecting a false peak with a power value of 2.13 . This is the probability of getting a $\chi^{2}$ value of $2.13 \times 174 \times 16$ or higher from a $\chi^{2}$ distribution with $2 \times 174 \times 16$ dof. This value is $3.8 \times 10^{-4}$. However, after securing the $0.3 \mathrm{~Hz}$ feature, we searched in two bins, one at $2 / 3$ and the other at $3 / 2$ (bin width of $0.125 \mathrm{~Hz}$ ). Considering the two trials, the significance in the fourth GTI is $2 \times 3.8 \times 10^{-4}$. This significance level does not take into account the number of GTIs searched yet. We estimate its global significance-considering all GTIs-in Section 3.1.5.

\subsubsection{ObsID 0693851201}

A feature at a frequency of $0.30 \pm 0.02 \mathrm{~Hz}$ (middle panel of Figure 4) as observed in the PDS of observation 0693850501 (taken roughly a week before this observation) was again present, albeit at a lower significance of $\gtrsim 3.3 \sigma$. Similar to the bottom-left panel of Figure 3, the dotted horizontal lines represent the 3 and the $4 \sigma$ confidence contours considering all frequency bins (trials) between 0.1 and $4 \mathrm{~Hz}$.

\subsubsection{Other Observations}

We then constructed PDS from each of the shorter observations. Observation 0150280401 showed evidence for a QPO with a centroid frequency of $0.46 \pm 0.02 \mathrm{~Hz}(\mathrm{rms}$ amplitude of $12 \pm 2 \%$ ), a value consistent with $3 / 2$ of $0.3 \mathrm{~Hz}$. The significance of this feature-again considering a search around $2 / 3$ and $3 / 2$ of $0.3 \mathrm{~Hz}$-is $2.8 \times 10^{-4}$ estimated as follows. First, we estimated the probability of getting a power value of 2.451 , i.e., the probability of getting a $\chi^{2}$ value of $2.451 \times 81 \times 4$ or higher from a $\chi^{2}$ distribution with
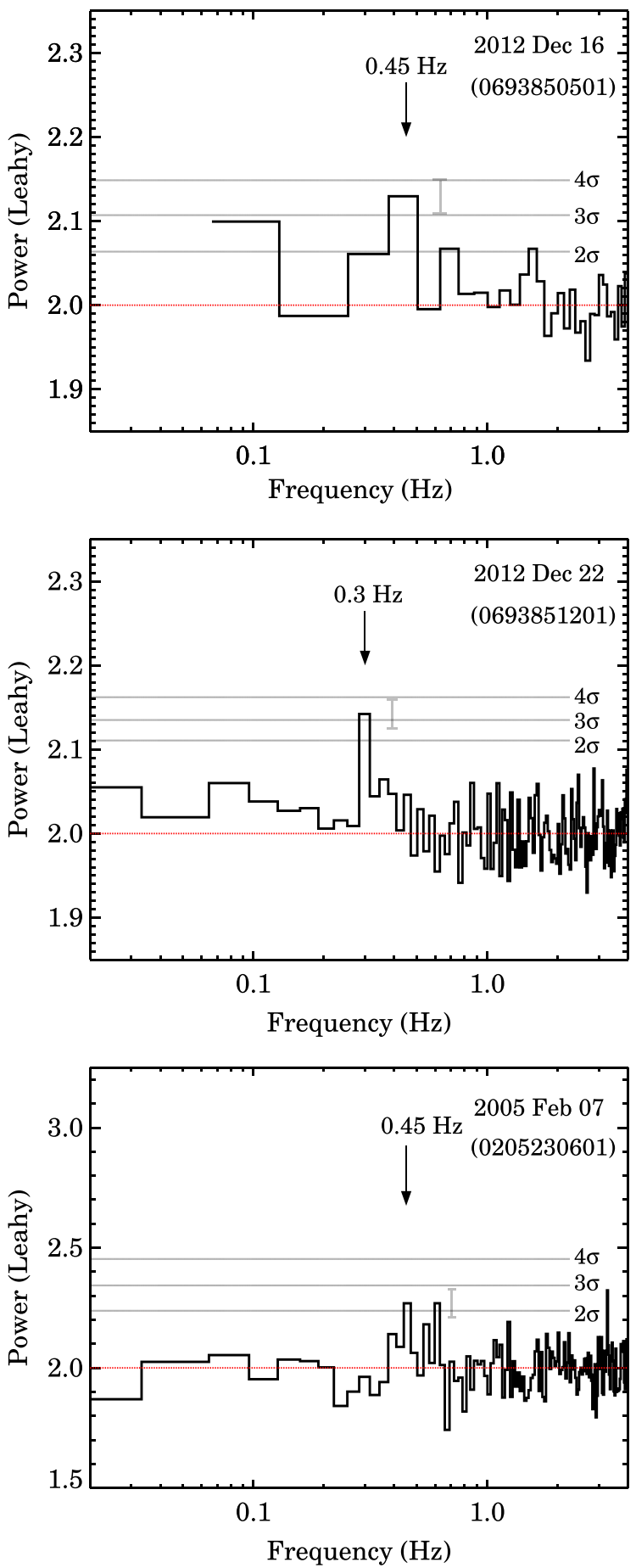

Figure 4. EPIC-pn $(0.3-10.0 \mathrm{keV})$ power spectra of NGC $1313 \mathrm{X}-1$ from observations 0693850501 (top: using only the last GTI of $\approx 22 \mathrm{ks}$ ), 0693851201 (middle), and 0205230601 (bottom). These show epochs-in addition to those shown in Figure 3-with excess power around 0.3 and $0.45 \mathrm{~Hz}$. The frequency resolution in the top, middle, and the bottom PDS is $0.125,0.03125$, and $0.03125 \mathrm{~Hz}$, respectively. The top PDS was constructed from $128 \mathrm{~s}$ light curve segments while the middle and the bottom were constructed from $256 \mathrm{~s}$ light curve segments binned at $1 / 8 \mathrm{~s}$. The number of PDS averaged in the top, middle, and bottom panels were 174,478 , and 48 , respectively. The confidence contours (estimated for a given observation and not global; see 3.1.5 for global significances) in the top and the bottom panels take into account two and four frequency bins, respectively (see text), while the contours in the middle panel take all the frequency bins between 0.1 and $4 \mathrm{~Hz}$ into account.

$2 \times 81 \times 4$ dof. This value is $7 \times 10^{-5}$. However, considering that we searched in two bins (width per bin of $0.03125 \mathrm{~Hz}$ ) around $3 / 2$ and $2 / 3$ of $0.3 \mathrm{~Hz}$, this translates to $4 \times 7 \times 10^{-5}$ 
$(>3.5 \sigma)$. The significance of the feature considering a full frequency search is $6 \times 10^{-3}$.

Observation 0205230601 showed weak evidence for a QPOlike feature at the $>2 \sigma$ level (bottom panel of Figure 4). The PDS from the remaining observations were essentially featureless with evidence for red noise in a handful of them. To ensure that these QPO features are not associated with the background, we extracted all the background PDS from these 15 observations. They are all consistent with being featureless (flat, white noise) within the error bars.

\subsubsection{Global Probability of the 0.45 and the $0.3 \mathrm{~Hz} Q P O s$}

In order to estimate the global significance of the $0.45 \mathrm{~Hz}$ QPO we considered all the 24 GTIs-longer than $7 \mathrm{ks}$ - where we searched for QPOs (see Table 1). Figures 3 and 4 show all the statistically significant QPOs detected during this search. The QPO's global probability can be calculated straightforwardly using the binomial distribution formula which gives the probability of happening of a certain event $m$ times in $n$ trials as

$$
P(m ; p, n)=\frac{n !}{m !(n-m) !} p^{m}(1-p)^{n-m}
$$

where $n$ is the total number of GTIs searched and $m$ is the number of GTIs where the signal was detected at a probability of $p$.

Using the above formula, the global probability of detecting the $0.45 \mathrm{~Hz}$ feature at $>7.6 \times 10^{-4}$ significance in $2(\mathrm{~m})$ out of the $24(n)$ GTIs is $1.6 \times 10^{-4}(>3.5 \sigma)$.

Similarly, we estimate the global probability of the $0.3 \mathrm{~Hz}$ QPO, using $n=24, m=1$, and $p=5.6 \times 10^{-7}(5 \sigma)$, to be $>3.5 \sigma$. Note that this is very conservative lower limit as we have not included the case where the $0.3 \mathrm{~Hz}$ feature was detected at $>3.3 \sigma$.

\section{DISCUSSION}

NGC 1313 X-1's PDS has all the features of a typical StMBH in the steep power-law state, but with characteristic timescales $\sim 1000 \times$ longer. Typical StMBHs have three components: (1) a continuum often flat-topped at the lowest frequencies with a power-law like decline beyond a certain break frequency followed by white noise at the highest frequencies, (2) low-frequency QPOs (frequencies of a few $\mathrm{Hz}$ ), and finally, (3) high-frequency QPOs (frequency range of $100-450 \mathrm{~Hz}$ ) exhibited by some systems. Three StMBHs with known masses show high-frequency QPOs in harmonic pairs with centroid frequencies in a ratio consistent with 3:2 (e.g., Miller et al. 2001; Strohmayer 2001a, 2001b, Remillard et al. 2002). In these systems, the two QPOs are often not simultaneous (e.g., see Table 1 of Remillard et al. 2002 and Strohmayer 2001b). Furthermore, unlike the low-frequency QPOs, these are stable in frequency with changes in source luminosity (McClintock \& Remillard 2006). Also, the timescales associated with them $(\sim 0.01 \mathrm{~s})$ are comparable to the Keplerian orbital periods of a test particle close to the innermost stable circular orbit. The commonalities of the high-frequency QPOs in these three StMBH systems suggest a common physical origin. Under the assumption that these QPOs originate from a radius fixed in gravitational units $(G M /$ $c^{2}$, where $G, M$, and $c$ are the gravitational constant, the black hole mass, and the speed of light, respectively), their frequency should simply scale inversely with the black hole mass. Indeed, the three StMBHs with high-frequency QPOs do agree with this inverse scaling law (see Figure 4.17 of McClintock \& Remillard 2006; Zhou et al. 2015).

We suggest that the observed lower 30 and $80 \mathrm{mHz}$ and the higher 0.3 and $0.45 \mathrm{~Hz}$ 3:2 ratio QPOs are the analogs of the low- and the high-frequency QPOs of StMBHs and that X-1 may be in an X-ray accretion state similar to the steep powerlaw state of StMBHs. This result agrees with prior work by Feng \& Kaaret (2006) who studied X-1's X-ray (0.3-10 keV) energy spectral variability using XMM-Newton data taken between 2000 and 2005. They concluded that the source resides in either the steep power-law state-at high luminosities - or in the low/hard state at lower luminosities, but never enters the thermal dominant state (see Bachetti et al. 2013 for alternate arguments).

Using the dynamical masses and the high-frequency QPOs of StMBHs ${ }^{7}$ XTE J1550-564, GRO J1655-50, and GRS 1915 +105 , we estimate - based on the inverse mass scaling - that $\mathrm{X}-1$ 's black hole mass is $5000 \pm 1300 M_{\odot}$. We also measured the mass using the break frequency-black hole mass-accretion rate scaling as derived by McHardy et al. (2006). Using a break frequency of $16 \pm 3 \mathrm{mHz}$ from obsID 0405090101 and assuming a lower limit on the bolometric luminosity of $2 \times 10^{40} \mathrm{erg} \mathrm{s}^{-1}$ implies a black hole mass of $>7600 \pm 5600$ $M_{\odot}{ }^{8}{ }^{8}$ This value is consistent with the measurement from the 3:2 QPO pair.

Yang et al. (2011) estimated X-1's bolometric correction factor (ratio of the X-ray to optical flux) to be similar to that of typical StMBHs, implying that a significant fraction of the emission is in the X-rays. X-1's average X-ray $(0.3-10.0 \mathrm{keV})$ luminosity of $2 \times 10^{40} \mathrm{erg} \mathrm{s}^{-1}$ (e.g., Feng \& Kaaret 2006) implies an Eddington ratio of $>0.03 \pm 0.01$. This value is significantly low compared to the typical Eddington ratios of StMBHs in the steep power-law state $\left(>0.2 L_{\text {Edd }}\right.$; McClintock $\&$ Remillard 2006). Assuming the 0.2 value reported by McClintock \& Remillard (2006), X-1's low Eddington ratio is inconsistent with the interpretation of it being in a steep powerlaw state.

Bachetti et al. (2013) carried out timing analysis of two of the data sets described here (0693850501 and 0693851201). However, they did not report any evidence for QPOs at $0.3 \mathrm{~Hz}$. We suspect the reason for this discrepancy is because they extracted their PDS using both the pn and the MOS data. As described earlier, MOS is limited to a frequency of $\approx 0.19 \mathrm{~Hz}$. In addition to any signal beyond $0.19 \mathrm{~Hz}$ being unreliable, even at frequencies lower than but close to $0.19 \mathrm{~Hz}$, signal suppression can be severe (van der Klis 1989). To test this, we extracted a PDS using a combined pn and MOS event list from the observations 0693850501 and 0693851201 . While we still see evidence for a feature at $0.3 \mathrm{~Hz}$ and at $\sim 90 \mathrm{mHz}$, this was statistically less significant than in the analysis presented above.

The first ULX 3:2 pair from M82X-1 was detected using the Rossi X-ray Timing Explorer $(R X T E)$, while the detection reported here is from XMM-Newton. Currently, XMM-Newton

\footnotetext{
XTE J1550-564, GRO J1655-50, and GRS 1915+105 have high-frequency QPOs at 184 and $276 \mathrm{~Hz}, 300$ and $450 \mathrm{~Hz}$, and 113 and $168 \mathrm{~Hz}$, respectively. Their black hole masses are $9.1 \pm 0.61 M_{\odot}$ (Orosz et al. 2011), $5.4 \pm 0.3 M_{\odot}$ (Beer \& Podsiadlowski 2002), and 10.1 $\pm 0.6 M_{\odot}$ (Steeghs et al. 2013), respectively.

8 The high uncertainty from the break frequency scaling is due to large error bars on the coefficients of the scaling law; see Figure 1 of McHardy et al. (2006).
} 
is the only X-ray observatory that can provide both a large enough effective area and the required time resolution to detect these oscillations from ULXs. Therefore, deeper X-ray observations of other variable ULXs, viz. NGC5408X-1 (Middleton et al. 2011) and NGC6946X-1 (Rao et al. 2010), are strongly encouraged. Also, the reported 3:2 pair from X-1 boosts confidence in the prospects of detecting high-frequency QPOs from relatively isolated ULXs with the Neutron star Interior Composition ExploreR (NICER)—with an anticipated effective area 1.5 times greater than that of EPIC-pn.

We thank the referee for valuable comments/suggestions that improved the paper.

\section{REFERENCES}

Abramowicz, M. A., Kluźniak, W., McClintock, J. E., \& Remillard, R. A. 2004, ApJL, 609, L63

Bachetti, M., Harrison, F. A., Walton, D. J., et al. 2014, Natur, 514, 202

Bachetti, M., Rana, V., Walton, D. J., et al. 2013, ApJ, 778, 163

Beer, M. E., \& Podsiadlowski, P. 2002, MNRAS, 331, 351

Begelman, M. C. 2002, ApJL, 568, L97

Belloni, T. M., Sanna, A., \& Méndez, M. 2012, MNRAS, 426, 1701

Cseh, D., Grisé, F., Kaaret, P., et al. 2013, MNRAS, 435, 2896

Dheeraj, P. R., \& Strohmayer, T. E. 2012, ApJ, 753, 139

Farrell, S. A., Webb, N. A., Barret, D., Godet, O., \& Rodrigues, J. M. 2009, Natur, 460, 73

Feng, H., \& Kaaret, P. 2006, ApJL, 650, L75

Gladstone, J. C., Copperwheat, C., Heinke, C. O., et al. 2013, ApJS, 206, 14

Gladstone, J. C., Roberts, T. P., \& Done, C. 2009, MNRAS, 397, 1836

Kaaret, P., Prestwich, A. H., Zezas, A., et al. 2001, MNRAS, 321, L29

Kaaret, P., Simet, M. G., \& Lang, C. C. 2006, Sci, 311, 491

King, A. R., Davies, M. B., Ward, M. J., Fabbiano, G., \& Elvis, M. 2001, ApJL, 552, L109

Körding, E. G., Migliari, S., Fender, R., et al. 2007, MNRAS, 380, 301

Leahy, D. A., Darbro, W., Elsner, R. F., et al. 1983, ApJ, 266, 160

Liu, J.-F., Bregman, J. N., Bai, Y., Justham, S., \& Crowther, P. 2013, Natur, 503,500
Matsumoto, H., Tsuru, T. G., Koyama, K., et al. 2001, ApJL, 547, L25

McClintock, J. E., \& Remillard, R. A. 2006, in Compact Stellar X-ray Sources, ed. W. Lewin \& M. van der Klis (Cambridge: Cambridge Univ. Press), 157

McHardy, I. M., Koerding, E., Knigge, C., Uttley, P., \& Fender, R. P. 2006, Natur, 444, 730

Mezcua, M., Roberts, T. P., Lobanov, A. P., \& Sutton, A. D. 2015, MNRAS, 448,1893

Middleton, M. J., Roberts, T. P., Done, C., \& Jackson, F. E. 2011, MNRAS, 411,644

Miller, J. M., Fabbiano, G., Miller, M. C., \& Fabian, A. C. 2003, ApJL, 585, L37

Miller, J. M., Fabian, A. C., \& Miller, M. C. 2004, ApJL, 614, L117

Miller, J. M., Walton, D. J., King, A. L., et al. 2013, ApJL, 776, L36

Miller, J. M., Wijnands, R., Homan, J., et al. 2001, ApJ, 563, 928

Motch, C., Pakull, M. W., Soria, R., Grisé, F., \& Pietrzyński, G. 2014, Natur, 514, 198

Orosz, J. A., Steiner, J. F., McClintock, J. E., et al. 2011, ApJ, 730, 75

Pasham, D. R., \& Strohmayer, T. E. 2013, ApJ, 771, 101

Pasham, D. R., Strohmayer, T. E., \& Mushotzky, R. F. 2014, Natur, 513, 74

Protassov, R., van Dyk, D. A., Connors, A., Kashyap, V. L., \& Siemiginowska, A. 2002, ApJ, 571, 545

Rao, F., Feng, H., \& Kaaret, P. 2010, ApJ, 722, 620

Remillard, R. A., McClintock, J. E., Orosz, J. A., \& Levine, A. M. 2006, ApJ, 637, 1002

Remillard, R. A., Muno, M. P., McClintock, J. E., \& Orosz, J. A. 2002, ApJ, 580, 1030

Roberts, T. P., Gladstone, J. C., Goulding, A. D., et al. 2011, AN, 332, 398

Ryder, S. D., Staveley-Smith, L., Malin, D., \& Walsh, W. 1995, AJ, 109, 1592

Steeghs, D., McClintock, J. E., Parsons, S. G., et al. 2013, ApJ, 768, 185

Strohmayer, T. E. 2001a, ApJL, 552, L49

Strohmayer, T. E. 2001b, ApJL, 554, L169

Tao, L., Feng, H., Grisé, F., \& Kaaret, P. 2011, ApJ, 737, 81

Timmer, J., \& Koenig, M. 1995, A\&A, 300, 707

Tombesi, F., Cappi, M., Reeves, J. N., et al. 2010, A\&A, 521, A57

van der Klis, M. 1989, in Timing Neutron Stars, ed. H. Ögelman \& E. P. J. van den Heuvel (New York: Kluwer Academic), 27

Vaughan, S., \& Uttley, P. 2005, MNRAS, 362, 235

Yang, L., Feng, H., \& Kaaret, P. 2011, ApJ, 733, 118

Zhou, X.-L., Yuan, W., Pan, H.-W., \& Liu, Z. 2015, ApJL, 798, L5

Zoghbi, A., Miller, J. M., Walton, D. J., et al. 2015, ApJL, 799, L24 